Research Article

\title{
Analysis of an M/PH/1 Retrial Queueing-Inventory System with Level Dependent Retrial Rate
}

\author{
Zaiming Liu, Xuxiang Luo $i$, and Jinbiao Wu \\ School of Mathematics and Statistics, Central South University, Changsha, Hunan, China \\ Correspondence should be addressed to Xuxiang Luo; luoxuxiang@csu.edu.cn
}

Received 28 March 2020; Revised 26 June 2020; Accepted 16 July 2020; Published 14 August 2020

Academic Editor: Sitek Paweł

Copyright (C) 2020 Zaiming Liu et al. This is an open access article distributed under the Creative Commons Attribution License, which permits unrestricted use, distribution, and reproduction in any medium, provided the original work is properly cited.

\begin{abstract}
We analyze a queueing-inventory system which can model airline and railway reservation systems. An arriving customer to an idle server joins for service immediately with exactly one item from inventory at the moment of service completion if there are some on-hand inventory, or else he accesses to a buffer of varying size (the buffer capacity varies and equals to the number of the items in the inventory with maximum size $S$ ). When the buffer overflows, the customer joins an orbit of infinite capacity with probability $p$ or is lost forever with probability $1-p$. Arrivals form a Poisson process, and service time has phase type distribution. The time between any two successive retrials of the orbiting customer is exponentially distributed with parameter depending on the number of customers in the orbit. In addition, the items have a common life time with exponentially distributed. Cancellation of orders is possible before their expiry and intercancellation times are assumed to be exponentially distributed. The stability condition and steady-state probability vector have been studied by Neuts-Rao truncation method using the theory of Level Dependent QuasiBirth-Death (LDQBD) processes. Several stationary performance measures are also computed. Furthermore, we provide numerical illustration of the system performance with variation in values of underlying parameters and analyze an optimization problem.
\end{abstract}

\section{Introduction}

In real life, people often purchase products for future use or cancel the purchased products due to some reasons. A typical case is that customers reserve flight/train/bus seats, in which the seats are regarded as inventory. When the fight/ train/bus departs, the inventory will be invalidated immediately, namely, the inventory products have common life time. In addition, some customers choose to cancel their order because of schedule changes, resulting in the increase of the inventory. Therefore, managers need to consider the above factors and formulate corresponding management policies to achieve the maximum economic benefits.

There is a growing research interest in various queueing systems with an attached inventory under different inventory management policies and lost sales. Here, we take a brief look at works carried out so far in some related context. A significant contribution for the development of queueinginventory system is [1], where stationary distribution of joint queue length and inventory process was derived in explicit product form for various $\mathrm{M} / \mathrm{M} / 1$-system with inventory under different inventory management policies. Schwarz and Daduna [2] investigated an $M / M / 1 / \infty$-system with inventory management, continuous review, exponentially distributed lead times, and backordering. They computed performance measures and derived optimality conditions under different order policies. Zhao and Lian [3] considered a queueing-inventory system with two classes of customers, and found a priority service rule to minimize the long-run expected waiting cost. Saffari et al. [4] researched an M/M/1 queueing system with inventory under $(r, Q)$ policy and lost sales, in which lead times are random variables. Krishnamoorthy et al. [5] considered a single server supply chain model with a $(r Q, K Q)$ production inventory system. Rejitha and Jose [6] considered a stochastic inventory model with positive service time and retrial of customers. A detailed review on queueing-inventory models with various cases can be seen in [7]. 
Retrial queueing system is widely used in many different fields such as modern communication system and supply chain management system. Artalejo [8] introduced the retry strategy into the stochastic inventory system for the first time and studied the numerical solution and optimal decision problem. On this basis, Ushakumari [9] studied a queueinginventory system with retrial and random lead time and obtained the optimal inventory ordering strategy $(s, S)$. However, the service time is often neglected in some reported queueing-inventory systems. In order to describe the actual situation, Berman et al. [10] considered positive service time firstly and formulated a model where both the demand and service rates are assumed to be constant and deterministic. Thereafter, Berman and Kim [11] extended the above model to the case where service time was a random variable. A discrete product inventory control system with positive service time was considered by Shajin et al. [12]. Krishnamoorthy et al. [13] made a detailed review of inventory models with positive service time and suggested possible directions for future research. One may refer this for the development taken place in queueing-inventory with retrial customers which contains some of the finest contributions such as [14-18].

Concepts of reservation, cancellation, and common life time was first introduced in the queueing-inventory system by Krishnamoorthy et al. [19] with a constant retrial rate. In their model, customers arrive according to a Poisson process and service time follows an exponentially distributed. Subsequently, they extended the above model to a $G I / M / 1$ type queueing-inventory systems with postponed work [20]. A queueing-inventory system with impatient customers and overbooking was analyzed by Shajin and Krishnamoorthy [21], in which customers arrive according to the Markovian arrival process and service time has phase type distribution. Furthermore, Shajin et al. [22] considered a single serve queueing-inventory system with facility for reservation in advance for the next $K$ time frames ahead. For more works on queueing-inventory models with reservation, cancellation, and common life time, we refer the reader to [23].

The aim of our research is to present stationary distribution and optimal inventory policies for service system with an attached inventory under reservation, cancellation of orders, common life time, and retrial. We analyze a single server queueing system of $M / P H / 1$-type with an attached inventory. Customers arrive according to a Poisson Process, and service time of each customer follows a $\mathrm{PH}$ distribution. The retrial times for any repeated customer are exponentially distributed. By rationing and canceling purchases, the inventory is replenished. In addition, we assume the items in the inventory have a common life time. Based on the above integrated model, we construct a multidimensional continuous time Markov chain, which is a Level Dependent Quasi-Birth-Death process (LDQBD). Note that the replenishment policies include rationing and cancellation of purchases, and common life time of all items is considered in our model, which is quite different from [6]. We provide the stationary probabilities and stationary condition using the theory of LDQBD. Several performance measures are calculated and their sensitivity to system parameters is analyzed. According to the calculation results, an optimization problem is discussed. By comprehensively analyzing the influence of various factors on the profit, it is illustrated that the change trend of the profit on the system parameters is in line with the actual situation, which provides an effective and scientific basis for the inventory manager.

\section{Model Description}

We consider an $\mathrm{M} / \mathrm{PH} / 1$ retrial queueing-inventory system with reservation, cancellation of orders, common life time, and retrial. The customers arrive according to a Poisson process with rate $\lambda$. The service time for each customer follows a $\mathrm{PH}$ distribution with representation $(\tau, \mathbf{T})$ of order $m$. It has the distribution function $B(x)=1-\tau e^{\mathrm{T} x} 1$, and the mean rate of service $\mu=\left[-\tau \mathbf{T}^{-1} 1\right]^{-1}$, where 1 is the column vector of 1's with appropriate order. There are $S$ items in the inventory at the beginning of a cycle. They have a common life time, which is exponentially distributed with parameter $\gamma$. If an arriving customer finds the server idle and there is at least one item in the inventory, the customer starts service immediately. At the moment of service completion, the customer takes exactly one item from the inventory away, and hence the on-hand inventory decreases by one. Otherwise, if an arriving customer finds the server busy, he joins a buffer of varying size depending on the number of items in the inventory (the buffer capacity varies and equals to the number of items in the inventory). If the buffer has reached the maximum capacity(that is, the number of customers in the buffer equals to the number of items in inventory), then an arriving customer either joins an orbit of infinite capacity with probability $p$ to seek the service again and again or leaves the system forever with complementary probability $1-p$. Inter-retrial times are exponentially distributed with rate $n \alpha$, when there are $n$ customers in the orbit. In addition, all customers in the buffer are assumed to leave the system when the common life time of the items is realized, and the customers in orbit do not leave the system on the contrary (that is, they are willing to wait for service in the next cycle). Thus, we define a cycle as the time duration from the epoch at which we start with $S$ items at a replenishment epoch, to the moment when the common life time is realized.

Two types of inventory replenishment policies are considered. One is the instantaneous replenishment (zero lead time) that the inventory reaches its maximum level $S$ on realization of common life time for the next cycle. The other is cancellation of orders (that is, returning of a sold item) before their expiry. It occurs according to an exponentially distributed interoccurrence time with parameter i $\beta$ when $(S-i)$ items in the inventory. By cancellation of purchases, inventory is increased until their expiry time. Note that inventory level will not go above $S$ by cancellation since the maximum possible number "in sold list" is $i$ when $(S-i)$ items are held in the inventory. Cancellation time is assumed to be negligible. Customers are served in FCFS discipline. The above processes are independent of each other. We start with the case of customers being flushed out from the finite buffer on realization of common life time. The graphical description of the proposed model is shown in Figure 1. 
Throughout this paper, we use the following notation: $N_{o}(t)=$ number of customers in the orbit at time $t$ $N_{b}(t)=$ number of customers in the buffer at time $t$ $N_{I}(t)=$ number of items in the inventory at time $t$ $J(t)=\left\{\begin{array}{l}0 \text { if serverisidleattimet } \\ j \text { if serverisbusyattimet and theservicephaseis } j\end{array}\right.$ $\mathbf{I}=$ identity matrix of appropriate size

$0=$ zero matrix of appropriate size

$K=S(S+3) / 2 m+S+1$

$K_{1}=(S-1)(S+2) / 2 m+S$

$K_{i}=1+(i+1) m, i=0, \ldots, S$

The process $\left\{\left(N_{o}(t), N_{I}(t), N_{b}(t), J(t)\right), t \geq 0\right\}$ is a continuous time Markov chain (CTMC), which is a LDQBD, with state space:

$$
\begin{array}{r}
\Omega=\{(n, i, 0,0) ; n \geq 0 ; 0 \leq i \leq S\} \\
\cup\left\{\left(n, i, n_{b}, j\right) ; n \geq 0 ; 1 \leq i \leq S, 0 \leq n_{b} \leq i ; 1 \leq j \leq m ;\right\} \\
\cup\{(n, S, 0) ; n \geq 0\} .
\end{array}
$$

The subset of the state space $\{(n, S, 0) ; n \geq 0\}$ consists of such states that the common life time is realized and the inventory level reaches its maximum $S$ in way of fresh replenishment with no customer in the buffer.

We enumerate the states of the above CTMC in lexicographic order. Thus, the infinitesimal generator matrix $\mathbf{Q}$ of this CTMC has the following structure:

$$
\mathbf{Q}=\left(\begin{array}{ccccc}
\mathbf{B}_{0} & \mathbf{A}_{0} & & & \\
\mathbf{C}_{1} & \mathbf{B}_{1} & \mathbf{A}_{1} & & \\
& \mathbf{C}_{2} & \mathbf{B}_{2} & \mathbf{A}_{2} & \\
& & \ddots & \ddots & \ddots
\end{array}\right) .
$$

The matrices $\mathbf{A}_{n}(n \geq 0), \mathbf{B}_{n}, \mathbf{C}_{n}(n \geq 1)$, and $\mathbf{B}_{0}$ are square matrices of order $K+1$ :

$$
\begin{aligned}
& \mathbf{A}_{n}=\left(\begin{array}{cc}
\widetilde{\mathbf{A}_{n}} & 0 \\
0 & 0
\end{array}\right), \\
& \mathbf{B}_{n}=\left(\begin{array}{cc}
\widetilde{\mathbf{B}_{n}} & \widetilde{\boldsymbol{\gamma}} \\
\tilde{\lambda \tau} & -(n \alpha+\lambda)
\end{array}\right), \\
& \mathbf{C}_{n}=\left(\begin{array}{cc}
\widetilde{\mathbf{C}_{n}} & 0 \\
\widetilde{n \alpha \tau} & 0
\end{array}\right), \\
& \mathbf{B}_{0}=\left(\begin{array}{cc}
\widetilde{\mathbf{B}_{0}} & \widetilde{\boldsymbol{\gamma}} \\
\tilde{\lambda \tau} & -\lambda
\end{array}\right),
\end{aligned}
$$

where $\widetilde{n \alpha \tau}=\left(0, \ldots, 0, n \tau \tau_{1}, \ldots, n \alpha \tau_{m}, 0, \ldots, 0\right) \quad$ and $\tilde{\lambda \tau}=\left(0, \ldots, 0, \lambda \tau_{1}, \ldots, \lambda \tau_{m}, 0, \ldots, 0\right)$ of dimension $K$ with $n \alpha \tau_{j}$ and $\lambda \tau_{j}(1 \leq j \leq m)$, respectively, at the $\left(K_{1}+1+j\right)^{t h}$ position and $\widetilde{\gamma}=\gamma 1$. For $0 \leq n_{b} \leq i$, denote the ordered sets:

$$
\left\langle n_{b}\right\rangle=\left\{\left(n_{b}, 1\right),\left(n_{b}, 2\right), \ldots,\left(n_{b}, m\right)\right\} .
$$

Then, the matrices $\widetilde{\mathbf{A}_{n}}$ and $\widetilde{\mathbf{C}_{n}}$ are square matrices of order $K$ which are defined by

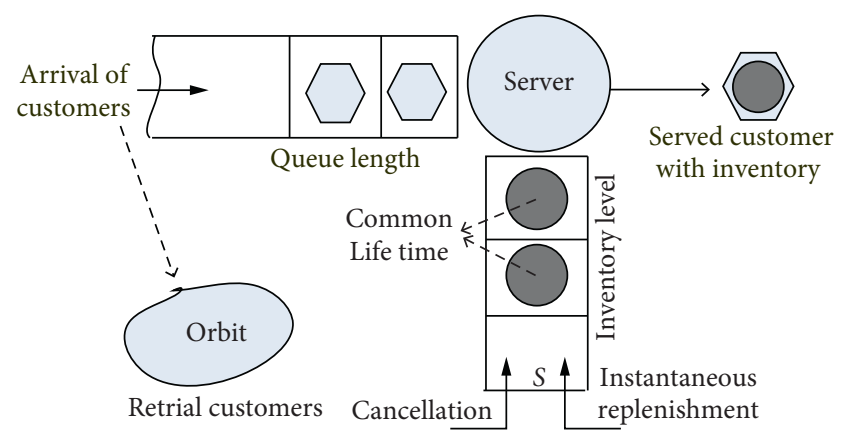

FIGURE 1: M/PH/1 queueing-inventory system with cancellation, common life time, and retrial.

$$
\begin{aligned}
& \widetilde{\mathbf{A}_{n}}=\operatorname{diag}\left(p \lambda, \mathbf{a}_{1}, \ldots, \mathbf{a}_{S}\right), \\
& \widetilde{\mathbf{C}_{n}}=\operatorname{diag}\left(0, \mathbf{c}_{1}, \ldots, \mathbf{c}_{S}\right),
\end{aligned}
$$

where

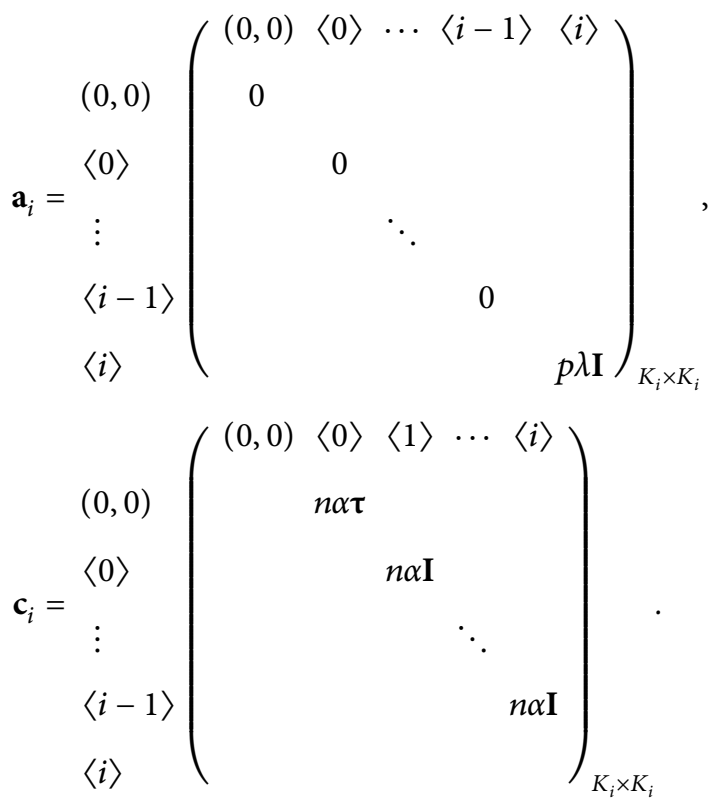

The matrices $\widetilde{\mathbf{B}_{0}}$ and $\widetilde{\mathbf{B}_{n}}$ of size $K \times K$ are represented by

$$
\widetilde{\mathbf{B}_{0}}=\left(\begin{array}{ccccc}
b_{0,0}^{0} & \mathbf{b}_{0,1} & & & \\
\mathbf{b}_{1,0} & \mathbf{b}_{1,1}^{0} & \mathbf{b}_{1,2} & & \\
& \ddots & \ddots & \ddots & \\
& & \mathbf{b}_{S-1, S-2} & \mathbf{b}_{S-1, S-1}^{0} & \mathbf{b}_{S-1, S} \\
& & & \mathbf{b}_{S, S-1} & \mathbf{b}_{S, S}^{0}
\end{array}\right) \text {, }
$$

$$
\widetilde{\mathbf{B}_{n}}=\left(\begin{array}{ccccc}
b_{0,0} & \mathbf{b}_{0,1} & & & \\
\mathbf{b}_{1,0} & \mathbf{b}_{1,1} & \mathbf{b}_{1,2} & & \\
& \ddots & \ddots & \ddots & \\
& & \mathbf{b}_{S-1, S-2} & \mathbf{b}_{S-1, S-1} & \mathbf{b}_{S-1, S} \\
& & & \mathbf{b}_{S, S-1} & \mathbf{b}_{S, S}
\end{array}\right) \text {, }
$$

where 


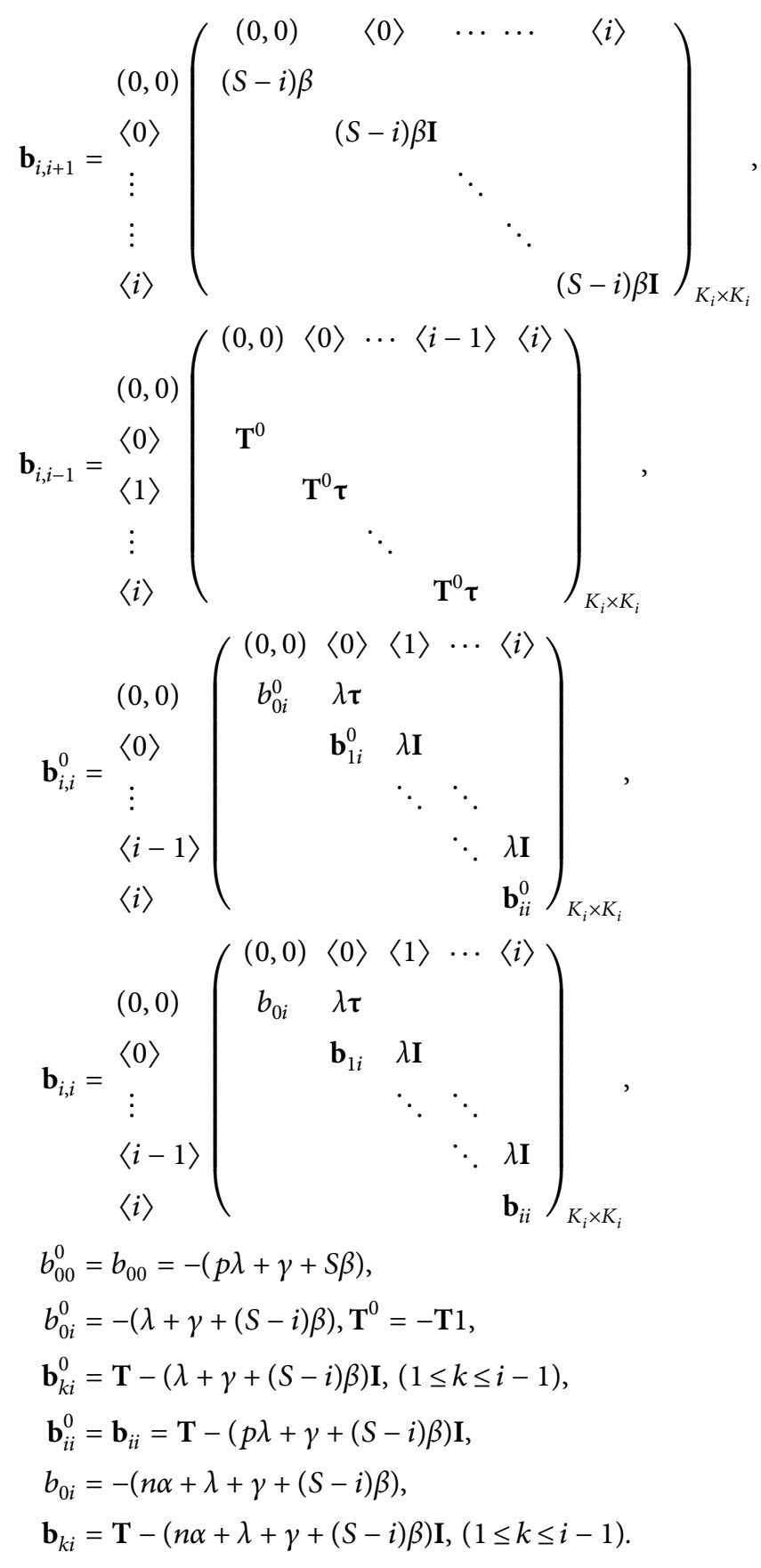

\section{Steady-State Analysis}

In this section, we study the stability condition of the queueing-inventory system and calculate the steadystate probability vector using the theory of LDQBD processes.

Denote the steady-state probabilities of the continuous time Markov chain as

$$
\begin{aligned}
P\left(n, i, n_{b}, j\right) & =\lim _{t \longrightarrow \infty} P\left\{N_{o}(t)=n, N_{I}(t)=i,\right. \\
N_{b}(t) & \left.=n_{b}, J(t)=j\right\} .
\end{aligned}
$$

Let $\pi$ denote the steady-state probability vector of the generator $\mathbf{Q}$. When the system is stable, we have

$$
\pi \mathbf{Q}=0, \pi 1=1
$$

Partitioning $\pi$ as $\pi=\left(\pi_{0}, \pi_{1}, \pi_{2}, \ldots\right)$, where each of the subvectors is denoted by

$$
\begin{array}{r}
\boldsymbol{\pi}_{n}=\left\{\boldsymbol{\pi}_{n}(S, 0)\right\} \\
\cup\left\{\boldsymbol{\pi}_{n}(i, 0,0) ; 0 \leq i \leq S\right\}
\end{array}
$$$$
\cup \pi_{n}\left\{\left(i, n_{b}, j\right) ; i, n_{b}, j ; 1 \leq i \leq S ; 0 \leq n_{b} \leq i ; 1 \leq j \leq m\right\} .
$$

Due to the multiple phases, it is hard to express $\pi$ in the closed form (e.g., generating function) even though $\mathbf{Q}$ is highly structured. In the following, we will adopt the Neuts-Rao truncation method using the theory of LDQBD processes to solve the system of equations given in (1). For more details about this theory, interested readers are referred to the book [24].

3.1. Stability Condition. Using the Neuts-Rao truncation method, we identify a positive integer $N$ suitably, such that defining $\mathbf{A}=\mathbf{A}_{\mathbf{n}}, \mathbf{B}=\mathbf{B}_{\mathbf{n}}$, and $\mathbf{C}=\mathbf{C}_{\mathbf{n}}$ for $n \geq N$, we have the following generator:

$$
\mathbf{Q}^{\prime}=\left(\begin{array}{cccccccc}
\mathbf{B}_{0} & \mathbf{A}_{0} & & & & & & \\
\mathbf{C}_{1} & \mathbf{B}_{1} & \mathbf{A}_{1} & & & & & \\
& \mathbf{C}_{2} & \mathbf{B}_{2} & \mathbf{A}_{2} & & & & \\
& & \ddots & \ddots & \ddots & & & \\
& & & \mathbf{C}_{N-1} & \mathbf{B}_{N-1} & \mathbf{A}_{N-1} & & \\
& & & & \mathbf{C} & \mathbf{B} & \mathbf{A} & \\
& & & & & \ddots & \ddots & \ddots
\end{array}\right) .
$$

Let $\boldsymbol{\pi}^{\prime}$ be the steady probability vector of $\mathbf{H}=\mathbf{A}+\mathbf{B}+\mathbf{C}$ :

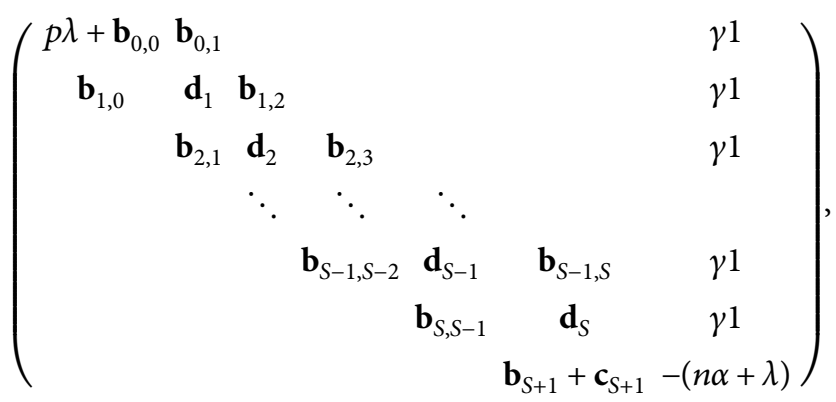

where $\quad \mathbf{d}_{i}=\mathbf{a}_{i}+\mathbf{c}_{i}+\mathbf{b}_{i, i}, 1 \leq i \leq S, \quad \mathbf{b}_{S+1}=(0, \lambda \tau, 0, \ldots)$, $\mathbf{c}_{S+1}=(0, n \alpha \tau, 0, \ldots)$, and $a_{0}=p \lambda$.

Then,

$$
\pi^{\prime} \mathbf{H}=0, \pi^{\prime} 1=1 .
$$

The components of $\pi^{\prime}$ are obtained as

$\pi_{i}^{\prime}=\pi_{i+1}^{\prime} \Phi_{i}^{\prime}, \quad$ for $0 \leq i \leq S$,

where 


$$
\boldsymbol{\Phi}_{i}^{\prime}= \begin{cases}-\mathbf{b}_{1,0}\left(p \lambda+b_{0,0}\right)^{-1}, & \text { for } \quad i=0, \\ -\mathbf{b}_{i+1, i}\left(\boldsymbol{\Phi}_{i-1}^{\prime} \mathbf{b}_{i-1, i}+\mathbf{d}_{i}\right)^{-1}, & \text { for } \quad 1 \leq i \leq S-1, \\ -\left(\mathbf{b}_{S+1}+\mathbf{c}_{S+1}\right)\left(\boldsymbol{\Phi}_{S-1}^{\prime} \mathbf{b}_{S-1, S}+\mathbf{d}_{S}\right)^{-1}, & \text { for } \quad i=S .\end{cases}
$$

From the equation,

$$
\left(\pi_{0}^{\prime}+\pi_{1}^{\prime}+\cdots+\pi_{S}^{\prime}\right) \gamma 1+\pi_{S+1}^{\prime}(-(n \alpha+\lambda))=0,
$$

and the normalizing condition $\pi^{\prime} 1=1$, we have

$$
\pi_{S+1}^{\prime}=\frac{\gamma}{n \alpha+\lambda+\gamma}
$$

The stability condition of the queueing-inventory system under study is given by the following theorem.

Theorem 1. The queueing-inventory system under study is stable if and only if

$$
\boldsymbol{\pi}_{S+1}^{\prime}\left(\sum_{k=0}^{S} \prod_{i=k}^{S} \boldsymbol{\Phi}_{i}^{\prime} \mathbf{a}_{i}\right) 1<\boldsymbol{\pi}_{S+1}^{\prime}\left(\sum_{k=1}^{S} \prod_{i=k}^{S} \boldsymbol{\Phi}_{i}^{\prime} \mathbf{c}_{i}+\boldsymbol{\Phi}_{S}^{\prime} \mathbf{c}_{S+1}\right) 1 .
$$

Proof. The queueing-inventory system in the model with the $L I Q B D$ type generator given in (2) is stable if and only if (see [25])

$$
\pi^{\prime} \mathrm{A} 1<\pi^{\prime} \mathrm{C} 1
$$

From the elements of $\mathbf{A}$ and $\mathbf{C}$, we have

$$
\boldsymbol{\pi}^{\prime} \mathbf{A} 1=\sum_{i=0}^{S} \boldsymbol{\pi}_{i}^{\prime} \mathbf{a}_{i} 1, \boldsymbol{\pi}^{\prime} \mathbf{C} 1=\sum_{i=1}^{S+1} \boldsymbol{\pi}_{i}^{\prime} \mathbf{c}_{i} 1
$$
(4)

Using (3) and (5), we acquire the stability condition

3.2. Steady-State Probability Vector. Assuming that the stability condition (4) is satisfied, we see that the steady-state probability vectors $\pi_{n}, n \geq N$ are given by [25]

$$
\boldsymbol{\pi}_{n+N-1}=\boldsymbol{\pi}_{N-1} \mathbf{R}^{n}, \quad \text { for } n \geq 1,
$$

where $\mathbf{R}$ is the minimal nonnegative solution of the matrix quadratic equation:

$$
\mathbf{A}+\mathbf{R B}+\mathbf{R}^{2} \mathbf{C}=0,
$$

and the vectors $\pi_{k}, 0 \leq k \leq N-1$ are obtained by solving the following equations:

$$
\begin{array}{r}
\boldsymbol{\pi}_{0} \mathbf{B}_{0}+\pi_{1} \mathbf{C}_{0}=0, \\
\boldsymbol{\pi}_{0} \mathbf{A}_{0}+\boldsymbol{\pi}_{1} \mathbf{B}_{1}+\boldsymbol{\pi}_{2} \mathbf{C}_{2}=0, \\
\pi_{k-1} \mathbf{A}_{k-1}+\boldsymbol{\pi}_{k} \mathbf{B}_{k}+\pi_{k+1} \mathbf{C}_{k+1}=0,2 \leq k \leq N-2, \\
\boldsymbol{\pi}_{N-2} \mathbf{A}_{N-2}+\boldsymbol{\pi}_{N-1}\left(\mathbf{B}_{N-1}+\mathbf{R C}\right)=0 .
\end{array}
$$

The normalizing condition is

$$
\sum_{n=0}^{N-2} \pi_{k} 1+\pi_{N-1}(\mathbf{I}-\mathbf{R})^{-1} 1=1,
$$

where

$$
\boldsymbol{\pi}_{k}=\boldsymbol{\pi}_{0} \prod_{i=1}^{k} \Psi_{i}, \quad 1 \leq k \leq N-1
$$

with

$$
\Psi_{i}=\left\{\begin{array}{lll}
-\mathbf{A}_{0}\left(\mathbf{B}_{1}+\boldsymbol{\Psi}_{2} \mathbf{C}_{2}\right)^{-1}, & \text { for } \quad i=1, \\
-\mathbf{A}_{i-1}\left(\mathbf{B}_{i}+\Psi_{i+1} \mathbf{C}_{i+1}\right)^{-1}, & \text { for } & 2 \leq i \leq N-2, \\
-\mathbf{A}_{N-2}\left(\mathbf{B}_{N-1}+\mathbf{R C}\right)^{-1}, & \text { for } & i=N-1 .
\end{array}\right.
$$

\section{Performance Measures}

In this section, we derive some stationary performance measures and specific probabilities descriptions for the inventory system.

(1) Expected number of customers in the orbit:

$$
E_{O}=\sum_{n=1}^{\infty} n\left[\pi_{n}(S, 0)+\sum_{i=0}^{S} \pi_{n}(i, 0,0)+\sum_{i=1}^{S} \sum_{n_{b}=0}^{i} \sum_{j=1}^{m} \pi_{n}\left(i, n_{b}, j\right)\right] .
$$

(2) Expected number of customers in the buffer:

$$
E_{B}=\sum_{n=0}^{\infty} \sum_{i=1}^{S} \sum_{n_{b}=1}^{i} \sum_{j=1}^{m} n_{1} \pi_{n}\left(i, n_{b}, j\right) .
$$

(3) Expected number of items in the inventory before realization of common life time:

$E_{I}=\sum_{n=0}^{\infty}\left[\sum_{i=1}^{S} i \pi_{n}(i, 0,0)+\sum_{i=1}^{S} \sum_{n_{b}=0}^{i} \sum_{j=1}^{m} i \pi_{n}\left(i, n_{b}, j\right)\right]$.

(4) Expected cancellation rate before realization of common life time:

$$
\begin{aligned}
E_{C R}= & \sum_{n=0}^{\infty}\left[\sum_{i=0}^{S-1}(S-i) \beta \pi_{n}(i, 0,0)\right. \\
& \left.+\sum_{i=1}^{S-1} \sum_{n_{b}=0}^{i} \sum_{j=1}^{m}(S-i) \beta \pi_{n}\left(i, n_{b}, j\right)\right] .
\end{aligned}
$$

(5) Expected purchase rate before realization of common life time:

$$
E_{P R}=\sum_{n=0}^{\infty} \sum_{i=1}^{S} \sum_{n_{b}=0}^{i} \sum_{j=1}^{m} T_{j}^{0} \pi_{n}\left(i, n_{b}, j\right),
$$

where $T_{j}^{0}(1 \leq j \leq m)$ represents the transfer rate from state $j$ to absorbing state $m+1$.

(6) Expected number of items in the inventory at the moment on realization of common life time (that is 
the lost items in inventory when the next cycle start-a typical case is flights/trains/buses departing with one or more vacant seats):

$$
\begin{aligned}
E_{L I}= & \sum_{i=1}^{S} i\left[p_{1}^{(i)} \pi_{0}(i, 0,0)+\sum_{j=1}^{m} p_{3}^{(i)} \pi_{0}(i, i, j)\right. \\
& \left.+\sum_{n_{b}=0}^{i-1} \sum_{j=1}^{m} p_{4}^{(i)} \pi_{0}\left(i, n_{b}, j\right)\right] \\
& +\sum_{n=1}^{\infty} \sum_{i=1}^{S} i\left[p_{2}^{(i)} \pi_{n}(i, 0,0)+\sum_{j=1}^{m} p_{3}^{(i)} \pi_{n}(i, i, j)\right. \\
& \left.+\sum_{n_{b}=0}^{i-1} \sum_{j=1}^{m} p_{5}^{(i)} \pi_{n}\left(i, n_{b}, j\right)\right],
\end{aligned}
$$

where

$$
\begin{aligned}
& p_{1}^{(i)}=\frac{\gamma}{\lambda+\gamma+(S-i) \beta}, \\
& p_{2}^{(i)}=\frac{\gamma}{n \alpha+\lambda+\gamma+(S-i) \beta}, \\
& p_{3}^{(i)}=\frac{\gamma}{p \lambda+\gamma+T_{j}^{0}+T_{j .}+(S-i) \beta}, \\
& p_{4}^{(i)}=\frac{\gamma}{\lambda+\gamma+T_{j}^{0}+T_{j}+(S-i) \beta}, \\
& p_{5}^{(i)}=\frac{\gamma}{n \alpha+\lambda+\gamma+T_{j}^{0}+T_{j}+(S-i) \beta} .
\end{aligned}
$$

Here, $p_{1}^{(i)}$, for instance, represents the probability of realization of common life time before an arriving or a cancellation occurs when the system states are $\{(0, i, 0,0) ; 1 \leq i \leq S\}$.

(7) Probability that the inventory is full at the time of realization of common life time (all seats remain vacant at the time flight takes off):

$$
\begin{aligned}
& P_{I F}=p_{1}^{(S)} \pi_{0}(S, 0,0)+\sum_{j=1}^{m} p_{3}^{(S)} \pi_{0}(S, S, j)+\sum_{n_{b}=0}^{S-1} \sum_{j=1}^{m} p_{4}^{(S)} \pi_{0}\left(S, n_{b}, j\right) \\
& +\sum_{n=1}^{\infty}\left[p_{2}^{(S)} \pi_{n}(S, 0,0)+\sum_{j=1}^{m} p_{3}^{(S)} \pi_{n}(S, S, j)+\sum_{n_{b}=0}^{S-1} \sum_{j=1}^{m} p_{5}^{(S)} \pi_{n}\left(S, n_{b}, j\right)\right] .
\end{aligned}
$$

(8) Probability that the inventory is depleted at the time of realization of common life time (all seats are sold out at departure time):

$$
P_{I D}=p_{1}^{(0)} \pi_{0}(0,0,0)+\sum_{n=1}^{\infty} p_{2}^{(0)} \pi_{n}(0,0,0) .
$$

(9) Expected number of purchases before realization of common life time:

$$
E_{I P}=\frac{1}{\gamma} \sum_{n=0}^{\infty} \sum_{i=1}^{S} \sum_{n_{b}=0}^{i} \sum_{j=1}^{m} \frac{T_{j}^{0}}{\gamma+T_{j}^{0}} \pi_{n}\left(i, n_{b}, j\right) .
$$

(10) Expected number of cancellations before realization of common life time:

$$
\begin{aligned}
E_{I C}= & \frac{1}{\gamma} \sum_{n=0}^{\infty} \sum_{i=0}^{S-1} \frac{(S-i) \beta}{\gamma+(S-i) \beta} \pi_{n}(i, 0,0) \\
& +\frac{1}{\gamma} \sum_{n=0}^{\infty} \sum_{i=1}^{S-1} \sum_{n_{b}=0}^{i} \sum_{j=1}^{m} \frac{(S-i) \beta}{\gamma+(S-i) \beta} \pi_{n}\left(i, n_{b}, j\right) .
\end{aligned}
$$

\section{Numerical Examples}

In this section, we present some numerical results that show the system performance measures with variations in values of underlying parameters.

We assume that the parameters satisfied the stability condition (4) are set as the following: $S=2, \lambda=10, \alpha=4, \gamma=2, \beta=6$, and $p=0.5$. The $\mathrm{PH}$ type distribution of service time with representation $(\tau, \mathbf{T})$ is described by the matrices:

$$
\begin{aligned}
& \mathbf{T}=\left(\begin{array}{cc}
-18 & 5 \\
6 & -20
\end{array}\right) \\
& \boldsymbol{\tau}=\left(\begin{array}{ll}
0.3 & 0.7
\end{array}\right)
\end{aligned}
$$

Thus, the mean rate of service is $\mu=13.5802$.

Table 1 provides the joint stationary probabilities of the queueing-inventory system. It can be seen that the largest value of the marginal probabilities (lateral and longitudinal) are equal to 0.1790 and 0.5488 , respectively, indicating that there are no items in inventory and no repeated customers in orbit.

5.1. Effect of the Maximum Inventory Level $S$ on Various Performance Measures. From Table 2, we observe that with the increasing of the maximum inventory level $S$, the measures, such as expected number of customers in the buffer, expected number of items in the inventory, expected cancellation rate before realization of common life time, and expected number of items in the inventory at the moment on realization, are increased as expected. However, the expected number of customers in the orbit experiences a decline owing to the increased buffer size.

\subsection{Effect of the Arrival Rate $\lambda$ on Various Performance} Measures. Table 3 reveals that the expected number of customers in the orbit and in the buffer and expected cancellation rate before realization of common life time are increased when the arrival rate $\lambda$ increases, whereas the expected number of items in the inventory before realization of common life time increases first with $\lambda$ and then keeps going down, which may be caused by cancellation of orders. However, the expected number of items in the inventory 
TABLE 1: The stationary distribution of the queueing-inventory system with $S=2, \lambda=10, \alpha=4, \gamma=2, \beta=6$, and $p=0.5$.

\begin{tabular}{|c|c|c|c|c|c|c|c|c|c|}
\hline States/ $n$ & 0 & 1 & 2 & 3 & 4 & 5 & $\ldots$ & 9 & Sum \\
\hline$(0,0,0)$ & 0.0669 & 0.0534 & 0.0309 & 0.0155 & 0.0071 & 0.0031 & $\ldots$ & 0.0001 & 0.1790 \\
\hline$(1,0,0)$ & 0.1025 & 0.0451 & 0.0189 & 0.0076 & 0.0029 & 0.0011 & $\ldots$ & 0.0000 & 0.1788 \\
\hline$(1,0,1)$ & 0.0208 & 0.0084 & 0.0033 & 0.0012 & 0.0005 & 0.0002 & $\ldots$ & 0.0000 & 0.0345 \\
\hline$(1,0,2)$ & 0.0356 & 0.0148 & 0.0059 & 0.0023 & 0.0009 & 0.0003 & $\ldots$ & 0.0000 & 0.0599 \\
\hline$(1,1,1)$ & 0.0152 & 0.0112 & 0.0061 & 0.0029 & 0.0013 & 0.0005 & $\ldots$ & 0.0000 & 0.0374 \\
\hline$(1,1,2)$ & 0.0218 & 0.0156 & 0.0083 & 0.0039 & 0.0017 & 0.0007 & $\ldots$ & 0.0000 & 0.0524 \\
\hline$(2,0,0)$ & 0.0513 & 0.0169 & 0.0057 & 0.0019 & 0.0006 & 0.0002 & $\ldots$ & 0.0000 & 0.0767 \\
\hline$(2,0,1)$ & 0.0298 & 0.0098 & 0.0033 & 0.0011 & 0.0004 & 0.0001 & $\ldots$ & 0.0000 & 0.0446 \\
\hline$(2,0,2)$ & 0.0468 & 0.0160 & 0.0055 & 0.0019 & 0.0006 & 0.0002 & $\ldots$ & 0.0000 & 0.0712 \\
\hline$(2,1,1)$ & 0.0190 & 0.0073 & 0.0027 & 0.0010 & 0.0004 & 0.0001 & $\ldots$ & 0.0000 & 0.0306 \\
\hline$(2,1,2)$ & 0.0237 & 0.0093 & 0.0035 & 0.0013 & 0.0005 & 0.0002 & $\ldots$ & 0.0000 & 0.0386 \\
\hline$(2,2,1)$ & 0.0117 & 0.0081 & 0.0042 & 0.0019 & 0.0008 & 0.0003 & $\ldots$ & 0.0000 & 0.0273 \\
\hline$(2,2,2)$ & 0.0123 & 0.0083 & 0.0042 & 0.0019 & 0.0008 & 0.0003 & $\ldots$ & 0.0000 & 0.0280 \\
\hline$(2,0)$ & 0.0915 & 0.0320 & 0.0114 & 0.0040 & 0.0014 & 0.0005 & $\ldots$ & 0.0000 & 0.1411 \\
\hline Sum & 0.5488 & 0.2563 & 0.1140 & 0.0485 & 0.0198 & 0.0079 & $\ldots$ & 0.0001 & 1.0000 \\
\hline
\end{tabular}

TABLE 2: Effect of $S$ on various performance measures: fix $\lambda=10, \alpha=4, \gamma=2, \beta=6$, and $p=0.5$.

\begin{tabular}{cccccc}
\hline$S$ & $E_{O}$ & $E_{B}$ & $E_{I}$ & $E_{C R}$ & $E_{L I}$ \\
\hline 2 & 0.7827 & 0.2698 & 0.9968 & 2.1776 & 0.0761 \\
3 & 0.3469 & 0.3879 & 1.6862 & 4.0324 & 0.1290 \\
4 & 0.1764 & 0.4882 & 2.4487 & 5.0467 & 0.1873 \\
5 & 0.0944 & 0.5622 & 3.2512 & 5.4666 & 0.2482 \\
6 & 0.0516 & 0.6118 & 4.0719 & 5.6028 & 0.3101 \\
7 & 0.0285 & 0.6430 & 4.8999 & 5.6371 & 0.3721 \\
\hline
\end{tabular}

TABLE 3: Effect of $\lambda$ on various performance measures: fix $S=5, \alpha=4, \gamma=2, \beta=6$, and $p=0.5$.

\begin{tabular}{lccccc}
\hline$\lambda$ & $E_{O}$ & $E_{B}$ & $E_{I}$ & $E_{C R}$ & $E_{L I}$ \\
\hline 8 & 0.0316 & 0.3575 & 3.2270 & 4.6199 & 0.3054 \\
9 & 0.0569 & 0.4557 & 3.2435 & 5.0620 & 0.2741 \\
10 & 0.0944 & 0.5622 & 3.2512 & 5.4666 & 0.2482 \\
11 & 0.1472 & 0.6752 & 3.2535 & 5.8342 & 0.2267 \\
12 & 0.2180 & 0.7928 & 3.2527 & 6.1663 & 0.2084 \\
13 & 0.3098 & 0.9131 & 3.2504 & 6.4649 & 0.1926 \\
\hline
\end{tabular}

immediately on realization of common life time shows a decreasing trend, resulting from an increasing demand arrival.

\subsection{Effect of the Retrial Rate $\alpha$ on Various Performance} Measures. As shown in Table 4, we observe that an increase in retrial rate $\alpha$ makes a sharp decrease in expected number of customers in orbit and a mild decrease in expected cancellation rate before realization of common life time. The former is attributed to large number of customers in the orbit getting into the buffer as $\alpha$ increases. However, there is a slight increase in expected number of customers in the buffer and expected number of items in the inventory before realization of common life time as $\alpha$ increases. To sum up, the retrial rate $\alpha$ rarely affects the performance measures except the expected number of customers in orbit; this is true obviously.
TABle 4: Effect of $\alpha$ on various performance measures: fix $S=6, \lambda=11, \gamma=2, \beta=6$, and $p=0.5$.

\begin{tabular}{cccccc}
\hline$\alpha$ & $E_{O}$ & $E_{B}$ & $E_{I}$ & $E_{C R}$ & $E_{L I}$ \\
\hline 2 & 0.1419 & 0.7449 & 4.0831 & 6.0127 & 0.2849 \\
3 & 0.1047 & 0.7453 & 4.0837 & 6.0097 & 0.2846 \\
4 & 0.0859 & 0.7456 & 4.0841 & 6.0072 & 0.2846 \\
5 & 0.0745 & 0.7458 & 4.0845 & 6.0052 & 0.2847 \\
6 & 0.0669 & 0.7460 & 4.0848 & 6.0034 & 0.2848 \\
7 & 0.0613 & 0.7461 & 4.0851 & 6.0019 & 0.2849 \\
\hline
\end{tabular}

5.4. Effect of Variation in $\gamma$ on $P_{I F}, P_{I D}, E_{I P}$, and $E_{I C}$. We assign the following values to the parameters: $S=5, \lambda=11, \alpha=4$, and $p=0.7$. Keeping $\beta$ fixed, the effect of $\gamma$ on measures $P_{I F}, P_{I D}, E_{I P}$, and $E_{I C}$ are depicted through Figure 2. As shown in Figure 2(a), we observe that the probability for the inventory being full at the time of realization of common life time (at $S$ ) increases sharply as $\gamma$ increases. Similarly, the probability for the inventory being depleted (at 0 ) decreases slowly with increasing value of $\gamma$. It can be seen that common life time $\gamma$ has a great influence on the probability $P_{I F}$ but almost does not affect the probability $P_{I D}$. The results accord with the expectations. Figure 2(b) shows that both expected number of purchases and expected number of cancellations before realization of common life time decrease with increasing value of $\gamma$ for fixed $\beta$. This is attributed to faster realization of common life time.

5.5. Effect of Variation in $\beta$ on $P_{I F}, P_{I D}, E_{I P}$, and $E_{I C}$. We assign the same values as above. Keeping $\gamma$ fixed, the effect of $\beta$ on measures $P_{I F}, P_{I D}, E_{I P}$, and $E_{I C}$ are displayed in Figure 3. Probability for the inventory being full at the time of realization of common life time (at $S$ ) is a linearly increasing function of $\beta$ (see Figure 3(a)). Conversely, the increase in $\beta$ results in a decrease in probability for the inventory being zero (at 0 ) at the time of realization of common life time. The former phenomenon can be explained that the set of states with inventory level at the maximum $S$ could be revisited several times due to cancellations in a cycle. The latter is due to the adding inventory as the value $\beta$ increases. Figure 3(b) 


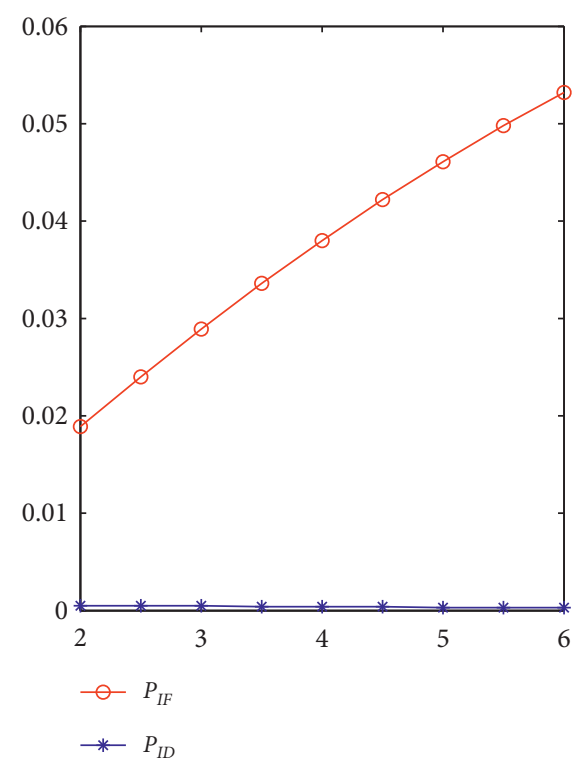

(a)

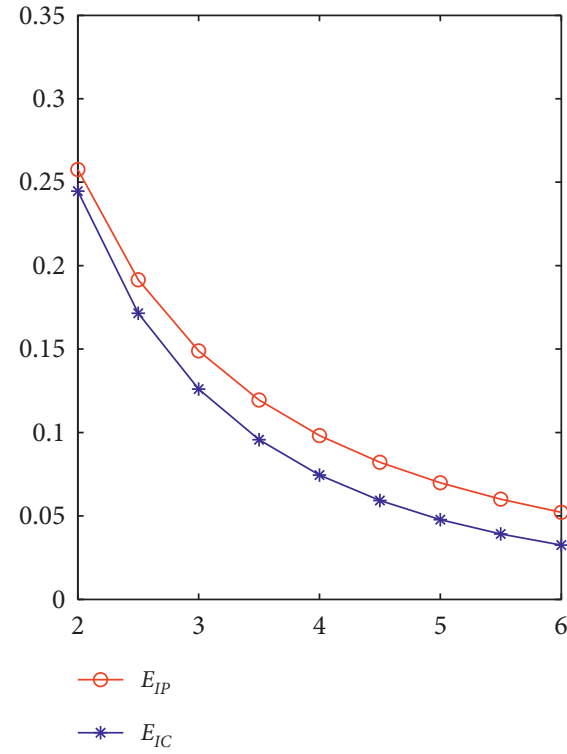

(b)

Figure 2: Effect of variation in $\gamma$ on $P_{I F}, P_{I D}, E_{I P}$, and $E_{I C}$ for $\beta=5$. (a) Effect of variation in $\beta$ on $P_{I F}$ and $P_{I D}$. (b) Effect of variation in $\beta$ on $E_{I P}$ and $E_{I C}$.

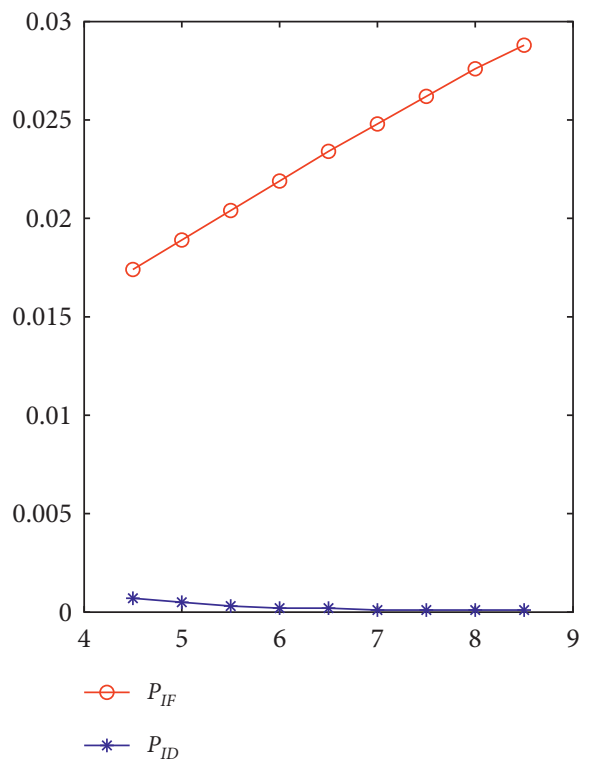

(a)

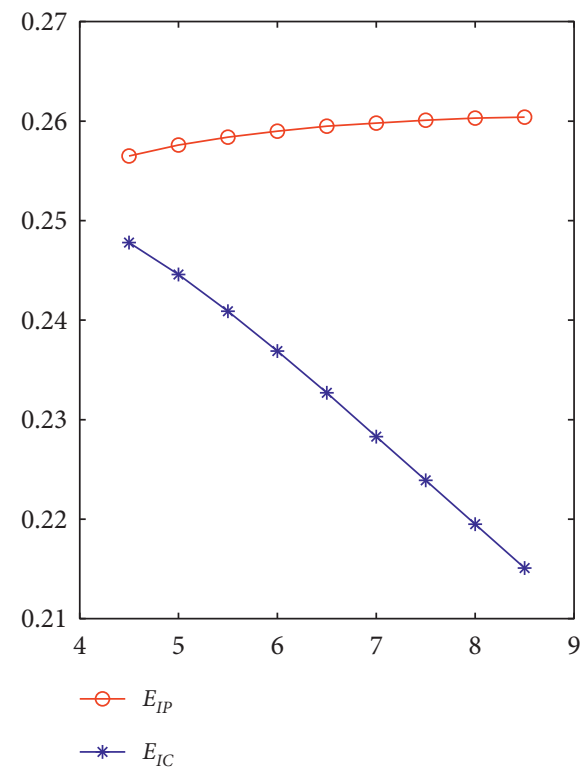

(b)

Figure 3: Effect of variation in $\beta$ on $P_{I F}, P_{I D}, E_{I P}$, and $E_{I C}$ for $\gamma=2$. (a) Effect of variation in $\beta$ on $P_{I F}$ and $P_{I D}$. (b) Effect of variation in $\beta$ on $E_{I P}$ and $E_{I C}$.

indicates that an increase in $\beta$ makes expected number of purchases before realization of common life time increase. Meanwhile, it is strange that expected number of cancellations before realization of common life falls off. The reason accounting for this phenomenon can be expressed by the provided $E_{I C}$ in Section4. The value of $E_{I C}$ depends on the multiplication factor and the probabilities. The former decreases as $i$ increases. On the contrary, the probabilities are risen rapidly with increasing value of $i$. Thus, their combined effect leads to a decline in the $E_{I C}$ value.

\section{Optimization}

Based on the above performance measures, we construct a cost function for checking the optimality of the inventory capacity $S$. It may be noted that the system profit will 
TABLE 5: Effect of $S$ on expected profit.

\begin{tabular}{lcccccc}
\hline$S$ & 2 & 3 & 4 & 5 & 6 & 7 \\
$F(S)$ & 36.6940 & 66.9920 & $\mathbf{7 8 . 8 4 9 5}$ & 77.4300 & 69.3315 & 58.5335 \\
\hline
\end{tabular}

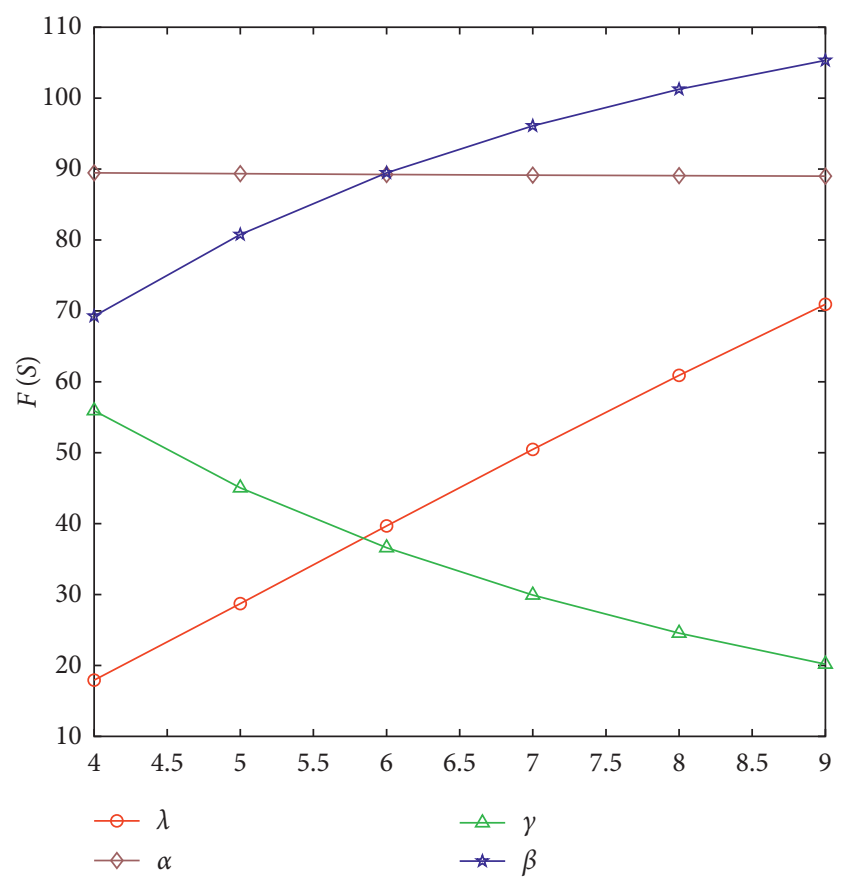

Figure 4: Effect of $\lambda, \alpha, \gamma$, and $\beta$ on expected profit.

increase when cancellation takes place before realization of common life time since there is a cancellation penalty imposed on the customer. Hence, we define profit function $F(S)$ as

$$
F(S)=c_{1} E_{C R}+c_{2} E_{B}-c_{3} E_{I}
$$

where $c_{1}$ represents the revenue to the system per unit cancellation of inventory purchased; $c_{2}$ represents the revenue to the system per customer per unit time in the buffer (this is an income provided by being sold items in account of inventory for sure); and $c_{3}$ represents the holding cost per unit time per inventory.

The objective of this section is to find the optimal inventory level $S$ that maximizes expected profit function $F(S)$ and analyze the effect of system parameters $\lambda, \alpha, \gamma$, and $\beta$, respectively, on expected profit. The cost coefficients are assumed that $c 1=$ per cancellation, $c 2=$ per customer per time, and $c 3=$ per item per time. Table 5 shows the optimal inventory level and the maximum profit when $\lambda=10, \alpha=4, \gamma=2, \beta=6$, and $p=0.5$.

In Table 5, we note that the optimal inventory size $S=4$, and the maximum expected profit is $\$ 78.8495$. Figure 4 presents expected profit with the changing of system parameters $\lambda, \alpha, \gamma$, and $\beta$, respectively, when other parameters are set the same as Table 5. In this figure, the expected profit is nondecreasing in $\lambda, \beta$ and nonincreasing in $\gamma$ as expected. Those phenomenons can be easily explained that there are more arriving demands and more inventory items due to the larger arrival rate and higher cancellation rate, respectively.
In addition, the shorter the common life time, the lesser the arriving demands and the number of cancellations, which results in the latter phenomenon. It is noteworthy that the retrial rate $\alpha$ has little impact on the expected profit. The appearance of this phenomenon may be based on the fact that the mean number of repeated customers is very small compared to the arriving customers.

\section{Conclusions}

This paper investigates a queueing-inventory system with reservation in advance, cancellation of orders, common life time, and retrial. Customers arrive according to a Poisson process and service time for each customer has phase type distribution. The time between any two successive retrials of the orbiting customer is distributed as an exponential with parameter depending on the number of customers in the orbit. We have constructed a multidimensional continuoustime Markov chain with infinitesimal generator which is level dependent. The stability condition and steady-state probability vector have been studied by the Neuts-Rao truncation method using $L D Q B D$. Several stationary performance measures have been obtained. In addition, we have established an optimization model for the inventory level and analyzed the effect of system parameters on various performance measures and expected profit.

This research can be generalized to the situation that each customer demands more than one unit items (one may order more than one ticket). Vacation policy and priority customers can also be introduced in this model.

\section{Data Availability}

The data used to support the findings of this study are included within the article.

\section{Conflicts of Interest}

The authors declare that they have on conflicts of interest.

\section{Acknowledgments}

This study was supported by the National Natural Science Foundation of China (Grant no.11671404).

\section{References}

[1] M. Schwarz, C. Sauer, H. Daduna, R. Kulik, and R. Szekli, "M/ M/1 queueing systems with inventory," Queueing Systems, vol. 54, no. 1, pp. 55-78, 2006.

[2] M. Schwarz and H. Daduna, "Queueing systems with inventory management with random lead times and with backordering," Mathematical Methods of Operations Research, vol. 64, no. 3, pp. 383-414, 2006.

[3] N. Zhao and Z. Lian, "A queueing-inventory system with two classes of customers," International Journal of Production Economics, vol. 129, no. 1, pp. 225-231, 2011.

[4] M. Saffari, S. Asmussen, and R. Haji, "The M/M/1 queue with inventory, lost sale, and general lead times," Queueing Systems, vol. 75, no. 1, pp. 65-77, 2013. 
[5] A. Krishnamoorthy, D. Shajin, and B. Lakshmy, "Product form solution for some queueing-inventory supply chain problem," Opsearch, vol. 53, no. 1, pp. 85-102, 2016.

[6] K. R. Rejitha and K. P. Jose, "A stochastic inventory system with two modes of service and retrial of customers," Opsearch, vol. 55, no. 1, pp. 134-149, 2018.

[7] K. Karthikeyan and R. Sudhesh, "Recent review article on queueing inventory systems," Research Journal of Pharmacy and Technology, vol. 9, no. 11, pp. 2056-2066, 2016.

[8] J. R. Artalejo, A. Krishnamoorthy, and M. J. Lopez-Herrero, "Numerical analysis of $(s, S)$ inventory systems with repeated attempts," Annals of Operations Research, vol. 141, no. 1, pp. 67-83, 2006.

[9] P. V. Ushakumari, "On $(s, S)$ inventory system with random lead time and repeated demands," Journal of Applied Mathematics and Stochastic Analysis, vol. 2006, pp. 1-22, 2006.

[10] O. Berman, E. H. Kaplan, and D. G. Shevishak, "Deterministic approximations for inventory management at service facilities"” IIE Transactions, vol. 25, no. 5, pp. 98-104, 1993.

[11] O. Berman and E. Kim, "Stochastic models for inventory management at service facilities," Communications in Statistics. Stochastic Models, vol. 15, no. 4, pp. 695-718, 1999.

[12] D. Shajin, B. Benny, R. V. Razumchik, and A. Krishnamoorthy, "Discrete product inventory control system with positive service time and two operation modes," Automation and Remote Control, vol. 79, no. 9, pp. 1593-1608, 2018.

[13] A. Krishnamoorthy, B. Lakshmy, and R. Manikandan, "A survey on inventory models with positive service time," Opsearch, vol. 48, no. 2, pp. 153-169, 2011.

[14] T. Yang and J. G. C. Templeton, "A survey on retrial queues," Queueing Systems, vol. 2, no. 3, pp. 201-233, 1987.

[15] B. K. Kumar, A. Vijayakumar, and D. Arivudainambi, “An M/ G/1 retrial queueing system with two-phase service and preemptive resume," Annals of Operations Research, vol. 113, no. 1-4, pp. 61-79, 2002.

[16] B. Sivakumar, "A perishable inventory system with retrial demands and a finite population," Journal of Computational and Applied Mathematics, vol. 224, no. 1, pp. 29-38, 2009.

[17] R. P. Hannah and M. G. Subramanian, "Two server $(s, S)$ inventory system with positive service time, positive lead time, retrial customers and negative arrivals," International Journal of Computer Applications, vol. 62, no. 10, pp. 9-13, 2013.

[18] T. Karthick, B. Sivakumar, and G. Arivarignan, "An inventory system with two types of customers and retrial demands," International Journal of Systems Science: Operations \& Logistics, vol. 2, no. 2, pp. 90-112, 2015.

[19] A. Krishnamoorthy, D. Shajin, and B. Lakshmy, "On a queueing-inventory with reservation, cancellation, common life time and retrial," Annals of Operations Research, vol. 247, no. 1, pp. 365-389, 2016.

[20] A. Krishnamoorthy, D. Shajin, and B. Lakshmy, "GI/M/1 type queueing-inventory systems with postponed work, reservation, cancellation and common life time," Indian Journal of Pure and Applied Mathematics, vol. 47, no. 2, pp. 357-388, 2016.

[21] D. Shajin and A. Krishnamoorthy, "On a queueing-inventory system with impatient customers, advanced reservation, cancellation, overbooking and common life time," Operational Research, vol. 47, pp. 1-25, 2019.

[22] D. Shajin, A. Krishnamoorthy, A. N. Dudin, V. C. Joshua, and V. Jacob, "On a queueing-inventory system with advanced reservation and cancellation for the next $\mathrm{K}$ time frames ahead: the case of overbooking," Queueing Systems, vol. 94, no. 1-2, pp. 3-37, 2020.

[23] D. Shajin, On Queueing-Inventory Models - Product Form Solution; Reservation, Cancellation and Common Life Time, Cochin University of Science and Technology, Kochi, Kerala, 2015.

[24] G. Latouche and V. Ramaswami, Introduction to Matrix Analytic Methods in Stochastic Modeling, Siam, New Delhi, India, 1999.

[25] M. F. Neuts, Matrix-geometric Solutions in Stochastic Models: An Algorithmic Approach, The Johns Hopkins University Press, Baltimore, ML, USA, 1981. 